\title{
ETHISCHE ASPEKTE DER PHYSIOTHERAPIE
}

\author{
Etické aspekty fyzioterapie
}

\section{Helmut Renöckl}

IX: 1 - 211, 2007

ISSN $1212-4117$

Kath. Theolog. Privatuniversität

Jihočeská univerzita v Českých Budějovicích, Teologická fakulta, katedra teologické etiky, sociální etiky a etického vzdělávání

\section{Souhrn}

Zdravotnická povolání a etika mají společné cíle: Při nezbytné specializaci musí mít vždy na zřeteli celého člověka a jeho kontext. Fyzioterapie je zaměřena na tělesné funkce, přičemž jsou stále více brány v úvahu souvislosti mezi svalovými, opěrnými a řídícími systémy. Kromě toho je nutno uvážit, že tělo a jeho potenciály nejsou jen něčím vnějším, ale že představují integrální dimenzi každého člověka, která uplatňuje mezi tělem, duchem a duší vzájemné komplexní interakce. Fyzioterapeuti se dotýkají lidí, kteří hledají uzdravení. Existují ozdravné, neužitečné i nesprávné doteky. Nezbytné jsou co nejlepší odborné znalosti a dovednosti a odpovídající osobní postoj.

Je pojednáno rovněž o vzrůstajícím napětí v trojúhelníku: lékařství - ekonomie - etika, je třeba vymezit otázky pojmů „kvalita“ a „účinnost“. Dále je naznačena nastupující změna paradigmatu v novověkých představách ve společnosti a v lékařství. Jasně se ukazuje slepá ulička iluzivní bezmeznosti a s tím spojeného individuálního a systémového přetěžování, zde nepomůže jednostranná technokratická a byrokratická maximalizace. Pomocí je však zesílené vznímání souvislostí, snaho o rovnováhu mezi tím, co je možné, a tím, co není $\mathrm{k}$ dispozici, jasné zaměření terapeutické činnosti na lidi hledající uzdravení, na obecné dobro a na zodpovědné nakládání s hranicemi.

Klíčová slova: etika terapeutických povolání, psychosomatika, životní styl, změna paradigmatu, lékařství - ekonomie - etika, účinnost, zajištění kvality, nakládání s hranicemi

\begin{abstract}
Heilende Berufe und Ethik haben gemeinsame Ziele: Bei aller notwendigen Spezialisierung muss immer der ganze Mensch und sein Kontext im Blick sein. Physiotherapie ist stark auf körperliche Funktionen ausgerichtet, wobei zunehmend die Zusammenhänge zwischen muskulären, stützenden und steuernden Systemen beachtet werden. Darüber hinaus ist zu bedenken, dass der Leib und seine Potentiale nichts bloß Äußerliches, sondern eine integrale Dimension jedes Menschen darstellen, dass sich zwischen Leib, Geist und Seele wechselseitig komplexe Interaktionen vollziehen. Physiotherapeuten greifen Heilung suchende Menschen an. Es gibt heilendes, nutzloses und übergriffiges Angreifen. Notwendig ist bestes fachliches Können und eine entsprechende persönliche Einstellung.

Erörtert werden auch die zunehmenden Spannungen im Dreieck Medizin - Ökonomie - Ethik, die Fragen, wie „Qualität“ und „Effizienz“ zu bestimmen sind. Weiters wird der spürbar in Gang kommende Paradigmenwechsel zu nachneuzeitlichen Vorstellungen in Gesellschaft und Medizin angesprochen. Die Sackgassen illusionärer Grenzenlosigkeit und die damit verbundenen individuellen und systemischen Überforderungen zeigen sich deutlich, einseitige technokratische und bürokratische Maximierungen führen nicht weiter. Hilfreicher sind eine verstärkte Beachtung der Zusammenhänge, das Bemühen um Balancen zwischen Machbarem und Unverfügbarem, eine klare Ausrichtung des therapeutischen Handelns auf Heilung suchende Menschen und das Gemeinwohl sowie ein verantwortlicher Umgang mit Grenzen.
\end{abstract}

Schlüsselwörter: Ethik therapeutischer Berufe, Psychosomatik, Lebensstil, Paradigmenwechsel, Medizin - Ökonomie - Ethik, Effizienz, Qualitätssicherung, Umgang mit Grenzen. 
Derzeit stehen das Medizinsystem und alle Menschen, die in diesem Bereich beruflich tätig sind, unter zunehmendem Druck. Die Medizinethik, wie ich sie vertrete, will diesen Druck nicht zusätzlich erhöhen. Es geht vielmehr um ein besseres Verstehen durch eine Erweiterung der Perspektiven, um hilfreiche Klärungen und Orientierungen. Das ermöglicht mehr innere Befriedigung und kann durch die Ausrichtung des Einsatzes auf angemessene Prioritäten und Proportionen den Druck sogar verringern.

\section{DIE ETHISCHE PERSPEKTIVE}

Zentrales Anliegen der Ethik ist die Grundfrage: „Wie gelingt unser Leben?“ Tragfähige Antworten auf diese Frage brauchen eine Zusammenschau vieler Bereiche und Perspektiven. Unser Leben gelingt weder automatisch, noch ein für alle Mal, sondern es entfaltet sich und gewinnt seine Gestalt in den vielen kleinen und gelegentlich großen Entscheidungen und Handlungen, samt den daraus erwachsenden Konsequenzen. Es geht der Ethik um ein bestmögliches Gelingen unseres Lebens als Ganzem, einschließlich der schönen und der schweren Stunden, sowie des persönlichen und des beruflichen Alltags. Schwere Stunden und Grenzsituationen sind ein entscheidender Test: Es gibt viele „Schönwetter"-Lebenskonzepte, die uns genau dann ohne Antwort, Orientierung und Halt lassen, wenn wir dies am dringendsten bräuchten. Den Kern der Ethik bilden nicht Vorschriften und Verbote, sondern unsere menschliche Denk- und Entscheidungsfähigkeit, unsere konstruktiven und destruktiven Potentiale und die mit unserem Denken und Handeln untrennbar verbundene Verantwortung.

Medizin/Therapie/Pflege und Ethik haben gemeinsame Ziele: Über alle Spezialisierungen hinaus geht es um den ganzen Menschen, um heilsames Leben, Gemeinwohl, Gesundheit der ganzen Bevölkerung, um die Kultivierung des Lebens und nicht nur um das „Reparieren“ von Erkrankungen und Verletzungen. Zum Leben der Menschen gehören immer die Umwelt und der gesellschaftliche Kontext. Mein Medizinethik-Ansatz ist grundsätzlich interdisziplinärdialogisch. Das heißt, ich bin nicht der Besserwisser im Bereich der Physiotherapie, sondern respektiere selbstverständlich die Fachkompetenzen und horche auf die von den Frauen und Männern, die in diesen Bereichen spezifisches Wissen und Können haben, geäußerten Erfahrungen, Fragen und Anliegen. Im interdisziplinären Diskurs finden sich am ehesten hilfreiche Klärungen und weiterführende Wege. Wie bei allen Berufsethiken in komplexen Systemen sind in der Medizinethik mehrere Ebenen zu beachten:

- Persönliche Einstellung/Haltung: Respekt, Wohlwollen, gegenüber Mitmenschen, Lebewesen und Werten

- Wissen - Können: Ziele - Wege/Mittel, Abschätzungen von Folgen und Risiken, Wirkungen und Nebenwirkungen, vorausgehend und begleitend

$\longrightarrow$ Persönliche Haltung und Praxis

$\triangle$ Strukturelle, institutionelle Regelungen, im eigenen Bereich, Berufsverbände, staatlich, überstaatlich

- Metahorizont: Glaube, Weltanschauung, epochale Paradigmen:

- Was ist der Mensch? Woher kommt er? Wohin geht er?

- Hoffnungen, Ängste, Motivationen, Antriebe,

- Orientierung, Werte, Prioritäten, Proportionen.

\section{DAS HEILSAME DER PHYSIOTHERAPIE}

Die Physiotherapie hat eine starke Ausrichtung auf die körperlichen Funktionen. Sie hilft verlorene bzw. eingeschränkte Beweglichkeit oder Stabilität wiederzugewinnen, nicht mehr gut
Koordinierbares wieder besser zu koordinieren, Verspanntes und Blockiertes zu lösen, in die richtigen Balancen zu bringen, Verkümmertes, Atrophiertes zu kräftigen ... Selbstverständlich macht man das heute unter Berücksichtigung 
der Zusammenhänge zwischen muskulären, stützenden, steuernden und versorgenden Systemen, wobei die Neuro- und die cerebrale Rehabilitation an Bedeutung zunehmen.

Der Leib und seine Potentiale sind nichts Äußerliches, sondern eine integrale Dimension jedes Menschen: Unser Wahrnehmen, Mitteilen, Kommunizieren und Handeln, unsere Energien, Ausdrucksformen und Abläufe, unsere Möglichkeiten und Grenzen haben ganz wesentlich eine leibliche Dimension. Zwischen Leib, Geist und Seele vollziehen sich fortwährend und wechselseitig komplexe Interaktionen. Hier setzt das physiotherapeutische Tun an, es geschieht in hohem Ausmaß hautnah, leibnah. Physiotherapeuten greifen Heilung suchende Menschen an. Es gibt heilendes und verletzendes, empathisches und desinteressiertes, gekonnt-hilfreiches, nutzloses und übergriffiges Berühren, Angreifen, Eingreifen. Es kommt in dieser leibhaften therapeutischen Beziehung viel darauf an, die richtige Balance zwischen empathischer Zuwendung und professioneller Distanz zu kennen und immer wieder $\mathrm{zu}$ finden. Wichtiges ist in beruflichen Verhaltensregeln/Guidelines fixiert, darüber hinaus geht es um eine angemessene persönliche Einstellung und Haltung, um die achtsame Balance von Fürsorge und Respekt vor der persönlichen Integrität und Verantwortlichkeit.

Selbstverständlich geht es bei einer fachlich wie ethisch guten Berufsausübung zuerst um die bestmögliche Durchführung einer konkreten Aufgabe, eines therapeutischen Auftrags. Physiotherapeuten haben keine medizinische Universalzuständigkeit. Zur professionellen Qualität gehört das Kennen und Einhalten von Kompetenzen. Es ist aber kein Luxus, Wissen und Sensibilität hinsichtlich möglicher tieferer Ursachen und Zusammenhänge der zu behandelnden Störung zu haben. Der Leib ist kein mit einfachen Handgriffen und anderen Instrumentarien reparierbarer Mechanismus. Wir wissen mittlerweile einiges über Psychosomatik, über krankmachende, verspannende, deformierende, Verkümmerungen bewirkende persönliche und gesellschaftliche Zusammenhänge und Lebensmuster. Wünschenswert wären eine über die unmittelbare Symptomatik hinausreichende Achtsamkeit der einzelnen therapeutischen Spezialisten und eine Verbesserung der noch unzureichend entwickelten Vernetzung aller an ei- nem Therapievorgang Beteiligten. Viele therapeutische Frustrationen bei Patienten und Therapeuten und erhebliche sinnlose Aufwände wären so zu vermeiden.

Im Rahmen jeder spezifischen Medizinethik (z. B. Ärzteethik, Pflegeethik, Physiotherapieethik, Psychotherapieethik) sind auch die schwierigen Herausforderungen im Dreieck Medizin - Ökonomie - Ethik, die aufklaffende Schere zwischen zunehmenden, meist teuren medizinischen Möglichkeiten und demgegenüber zurückbleibenden finanziellen Ressourcen zu thematisieren. Die Verteilungskämpfe um Ressourcen im Medizinbereich nehmen an Härte zu. Wer die reale Macht- und Geldverteilung, die Hauptakteure in diesem Bereich einigermaßen kennt, weiß, dass die Physiotherapeuten hier weder die großen Nutznießer, noch die BigPlayer an den Ressourcen-Steuerungs-Rädern sind. Allerdings sind in diesen Auseinandersetzungen proportional von allen Beteiligten neben berechtigten eigenen Interessen auch die Sachwalter-Funktion für die Patienten und die anteilige Mitverantwortung für einen optimalen Ressourcen-Einsatz im medizinischen Gesamtsystem zu beachten. Andernfalls treffen die negativen Konsequenzen unsinniger und ungerechter Ressourcen-Verteilung in unverantwortlichem Ausmaß die Patienten, die Schwächsten -, aber eigentlich die entscheidende, rechtfertigende Zielgruppe aller Aktivitäten im medizinischen System. Als ethische Leitlinie ist hier festzuhalten: „Vor Einschränkung notwendiger und sinnvoller medizinischer Leistungen muss eine Minimierung von Vergeudung und Nutzlosem angestrebt werden."

Spezielle ethische Fragen stellen sich hinsichtlich vergleichender therapeutischer Studien an Patienten. Wenn solche Studien an Zahl, Wirkungstiefe und Risiken zunehmen, dann empfiehlt sich die Einrichtung einer Physiotherapie-Ethik-Kommission (eine für das ganze Land) in Analogie zu den MedizinethikKommissionen. Für die Begutachtung von Studien halten die WHO-Richtlinien als Aufgabe der Ethik-Kommissionen fest: Die Würde, die Rechte, die Sicherheit und das Wohlergehen der Teilnehmer medizinischer Forschungsprogramme und die Glaubwürdigkeit der StudienErgebnisse sind zu sichern. Beides ist wichtig: die Rücksicht auf leidende Menschen in prekären, schutzbedürftigen Lagen und die wissen- 
schaftliche Qualität der Studien, geht es doch um Wirksamkeit, Risiken und Nebenwirkungen von Therapien und auch um deren Angemessenheit hinsichtlich Aufwand, Belastungen und Ergebnissen.

Das Ziel des physiotherapeutischen Einsatzes sind nachhaltig heilsame Ergebnisse für die Menschen. Dafür sind hohes spezifisches Fachwissen und optimal trainierte Fertigkeiten die Kernkompetenzen. Anstrebenswert sind zusätzlich ein fundierter Überblick über das unmittelbar Praktische hinaus und die daraus erwachsende Fähigkeit, den Sinn und Platz der eigenen Beiträge im größeren Zusammenhang verstehen und einordnen, sich anbahnende wünschenswerte oder problematische Entwicklungen im Fach und im relevanten Umfeld einschätzen und konstruktiv damit umgehen zu können. Gerade der Medizinbereich ist nicht isoliert zu verstehen, er manifestiert vielmehr wie ein Seismograph offensichtliche und hintergründige Vorgänge im persönlichen und gesellschaftlichen Leben.

\section{PARADIGMENWANDEL IN GESELLSCHAFT UND MEDIZIN}

Wir leben in einer Zeit rasanter und tiefgreifender Veränderungen in allen Lebensbereichen, gerade im medizinischen Bereich sind die Veränderungen gewaltig. Erinnern wir uns: Noch bis vor rund fünfzig Jahren waren die medizinischen Möglichkeiten beschränkt und für Ärzte wie Patienten überschaubar. Das medizinische Berufsethos, im Wesentlichen von Ärzten formuliert, wurde als angemessen und ausreichend empfunden. Seit den 1950er-Jahren wurden künstliche Beatmung, Reanimation und Lebenserhaltungssysteme eingeführt und zur ständig leistungsfähiger werdenden Intensivmedizin weiterentwickelt. 1954 gelang die erste Nierenverpflanzung; heute transplantiert man ganze Organpakete, operiert erfolgreich zerstörte Gelenke und replantiert abgetrennte Gliedmaßen, man operiert am Gehirn und an der Wirbelsäule. Die Palette hochwirksamer Pharmaka und Therapien wächst Jahr für Jahr weiter, man praktiziert pränatales und prädiktives Genscreening, über gentechnisch hergestellte Medikamente und Gendiagnostik stößt man zur molekularen Medizin vor. In diesem Zusammenhang erfolgten und erfolgen auch starke Erweiterun- gen der Einsatzfelder und Behandlungsformen der Physiotherapie.

Übersehen wir nicht: Diese rasante Expansion und Differenzierung des medizinischen Systems baut auf komplexe Finanzierungs- und Verwaltungssysteme, auf riesige, weltweit vernetzte, medizinische, pharmazeutische und medizintechnische Forschungs- und Produktionskomplexe, die ihre Eigengesetzlichkeiten und Dynamiken als bedeutsame Faktoren in das medizinische System einbringen. Wenn das Medizinsystem in den entwickelten Ländern rund $10 \%$ des Bruttoinlandsproduktes bewegt, so stimulieren diese ökonomischen Größenordnungen entsprechende Verteilungskämpfe, offen oder noch öfter verschleiert. In erheblichem Ausmaß schieben sich zusätzliche Akteure und Zusammenhänge, Mechanismen und Interessen zwischen Ärzte, Therapeuten, Pflegende und behandlungsbedürftige kranke Menschen. Es kommt zu Anonymisierungs- und Entfremdungstendenzen, zu System-Zwängen und hohen Aufwänden auf Kosten der personalen $\mathrm{Zu}-$ wendung, zu tiefgehenden Frustrationen bei den Heilung Suchenden ebenso wie bei den im Medizinsystem beruflich Tätigen.

Angesichts der Anspannung der finanziellen Ressourcen gibt es starke Trends zu Entsolidarisierung, zu neuem „Sozialdarwinismus“, zu technokratischen „Optimierungen“. Werden Gesundheit der Gesamtbevölkerung und Gemeinwohl nachrangig? Welchen Stellenwert hat in diesem Kontext der einzelne Heilung suchende Mensch? Selbstverständlich brauchen wir eine sorgfältige Kalkulation des Kalkulierbaren, korrekte Rechnungen, einen ökonomischen Umgang mit den begrenzten Ressourcen. „Ökonomisch" heißt, die verfügbaren Mittel und Verfahren optimal auf anstrebenswerte Ziele auszurichten. Das kann gerade nicht die Auflösung der Humanität in materielle Profitabilität oder grobe Vorteile bestimmter Gruppen auf Kosten anderer bedeuten. Besonders eindringlich muss in diesem Zusammenhang auf der unverfügbaren Würde jedes Menschen, unabhängig von Leistungsfähigkeit und Nützlichkeit, insistiert werden. Gesundheit ist nicht ein sachhaft herstellbares und handelbares Gut, das Produzenten an Konsumenten verkaufen. Patienten sind nicht souveräne Kunden, sondern Menschen in Notlagen, die sich nicht ausreichend selbst helfen können, vielmehr auf Hilfe 
angewiesen sind.

Es gibt nicht nur „äußerliche“ Anspannungen und Verspannungen, sondern auch hintergründige, ,innerliche“ Indizien für einen unausweichlichen Paradigmenwechsel: Die Leistungsfähigkeit der Medizin schafft neue, schwierige Situationen, welche die bisherigen Sichtweisen, Bewertungs- und Bewältigungsmuster der Medizin übersteigen: Durch die erweiterten diagnostischen und therapeutischen Möglichkeiten werden beispielsweise bei Frühgeburten die Grenzen immer weiter hinausgeschoben, man bringt extreme „Frühchen“ mit minimalen Geburtsgewichten und noch unreifen lebenswichtigen Organen durch, teils mit erfreulich guten Prognosen, zunehmend aber auch mit sehr schweren Behinderungen. Nach welchen Kriterien sind in solchen Lagen welche intensivmedizinischen, lebenserhaltenden Maßnahmen einzusetzen bzw. aus guten Gründen zu lassen? Neue Fragen stellen sich mitten im Leben: Ist jede Befindlichkeitsstörung eine behandlungsbedürftige Krankheit? Besteht Anspruch auf ununterbrochene „Wellness"? Wie sind „Verbesserungen“ der Menschen - therapeutische, chirurgische, hormonale, vielleicht einmal auch genetische $\mathrm{zu}$ bewerten? Ein anderes Beispiel: Soziologen konstatieren eine alternde Gesellschaft mit einer rasch zunehmenden Zahl hochbetagter, aufwendig pflegebedürftiger chronisch Kranker. Die starke Zunahme demenzkranker Hochbetagter wirft die Frage auf, ob die moderne Medizin die körperlichen Funktionen länger vital erhalten könne als die cerebralen Voraussetzungen für die geistigen Fähigkeiten. Man fragt immer öfter: Ist Lebensverlängerung um jeden Preis anzustreben, wird da mitunter nicht eher das Sterben als das Leben verlängert? Die Motivation solcher Fragen reicht von ehrlicher Empathie bis hin $\mathrm{zu}$ rücksichtslosem Egoismus. Vor "Patentantworten“ und kurzschlüssigen "Lösungen" in verschiedene Richtungen ist eindringlich $\mathrm{zu}$ warnen.

Offensichtlich herrscht im Unterschied zur früheren Überschaubarkeit der begrenzten Möglichkeiten und einer darauf aufbauenden Medizinethik jetzt ein hohes Maß von Unübersichtlichkeit hinsichtlich der Möglichkeiten und Grenzen, der Vorteile und Kosten, sowie hinsichtlich einer gerechten Verteilung der Ressourcen. In unserer spätmodernen Zivilisation werden über alle Kanäle der Massenkommuni- kation grenzenlose Versprechungen verbreitet und grenzenlose Erwartungen stimuliert: „Alles ist möglich", leistbar, kaufbar, und zwar „instant“!? Besteht Anrecht auf „Full Wellness, full Power for ever“, auf „Anti-Aging“? Maßlose Ansprüche der einen führen angesichts begrenzter Ressourcen zu Mangel an Notwendigem bei den anderen. Die rasante Zunahme von Wissen und Verfügungsmöglichkeiten ergeben neue Dilemmata und Entscheidungslagen sowohl bei wesentlichen Belangen der Lebensqualität als auch bei Entscheidungen über Leben oder Tod. Es gibt gefährliche Trends zu als „autonom“ bezeichneten, aber nicht verallgemeinerungsfähigen, sondern teils utilitaristisch-rücksichtslosen, teils individualistisch-willkürlichen Einstellungen, $\mathrm{zu}$ technokratischen oder sozialdarwinistischen „Lösungen“. In dieser Lage ist ein sorgfältiger Rückblick und Ausblick auf das uns Menschen Gemäße, auf das unverkürzte Ganze des Lebens unverzichtbar. Ohne Zweifel sind Sichtweisen und Antworten auf diese fundamentalen Fragen stark von epochalen Paradigmen geprägt.

\section{AKZENTE NEUZEITLICHER UND NACH-} NEUZEITLICHER MEDIZIN/THERAPIE

Die ganze Menschheitsgeschichte bis ins ausgehende Mittelalter herauf galt generell und in Bezug auf die Medizin das Gegebene/Überkommene im Wesentlichen als naturgemäß und gottgewollt, mit dem man sich daher, einschließlich Leid, Not und oft frühem Tod, im Großen und Ganzen abzufinden hatte. Das medizinische Tun bestand weitgehend in der Unterstützung der natürlichen Heilkräfte und in oft bewundernswerter Fürsorge und Pflege. Für intensivere Behandlungen und Eingriffe fehlten nicht nur wichtige Voraussetzungen, sondern sie galten weitgehend als unerlaubte Eigenmächtigkeiten gegen die Natur und die göttliche Ordnung. Ganz anders interpretierte die Neuzeit „die Natur" bzw. die überkommenen, sehr engen Lebensverhältnisse als Ausgangslage, als „Steinbruch“ menschlichen Gestaltens. Man betrieb daher aktiv die Erforschung der Wirklichkeit, um sie nach menschlichen Zielsetzungen umzugestalten. Konkret strebte man die Überwindung der engen Begrenzungen, die Beseitigung von Ohnmacht, Not und Leid an und glaubte, die umfassende Befreiung erreichen zu können. In Antithese zur bisherigen 
Orientierung waren dabei die Vorstellungen und Methoden der Naturwissenschaften leitend. So setzte man und setzt man bis heute vorrangig auf Spezialisierung und Quantifizierung, auf die messbaren physiologischen Indikatoren und die messbaren Wirkungen von Wirkstoffen und Behandlungen.

Das neuzeitliche Entgrenzungs-Programm brachte gewaltige Erfolge. Man denke nur an die verdoppelte durchschnittliche Lebenserwartung und an die für viele bis ins hohe Alter gute Lebensqualität. Zunehmend werden aber jetzt die Einseitigkeiten und offen bleibenden Fragen dieser Programmatik deutlich. Man vergleiche die WHODefinition: „Gesundheit ist ein Zustand vollständigen physischen, psychischen und sozialen Wohlbefindens und nicht einfach die Abwesenheit von Krankheit und Gebrechen. "Diese Definition war Leitlinie beim Aufbau der modernen Medizin-, Sozial- und Wohlfahrtseinrichtungen. Sie weckt aber auch grenzenlose Erwartungen, sie verschweigt und verdeckt besonders schwierige Realitäten des Lebens, wie die unausweichlichen Belastungen und Krisen, Grenzen und Schwächen, das Altern und Sterben. Diese einseitige Programmatik blockiert das Unterscheiden zwischen beseitigbarem und unvermeidlichem Leid und die Fähigkeit, unvermeidliche Belastungen und irreversible Einschränkungen auszuhalten und anzunehmen. Zusätzlich wird suggeriert, für alles gäbe es machbare, kaufbare Lösungen, alles wäre ,instant“, ,auf Knopfdruck“ zu bekommen, alles wäre jederzeit wiederholbar.

Mit solchen Illusionen macht man nicht nur unverschämte Geschäfte. Das Verdrängen der erwähnten schwierigen Lebensdimensionen führt $\mathrm{zu}$ individuellen und gesellschaftlichen Fehlentwicklungen und Schäden und verstärkt die Gerechtigkeitsprobleme: Die Durchsetzungsfähigeren beanspruchen im sinnlosen Übermaß auf Kosten anderer die Ressourcen. Und es wird dabei übersehen, dass Krankheit und Leiden nicht nur einen bedrückend dunklen Charakter, sondern persönlich und gesellschaftlich auch eine ent-täuschende, klärende, „prophetische“ Funktion haben können. Vielfach werden wir in unserer gefährlicheinseitigen Zivilisation erst durch Kranksein zur „Entschleunigung“ und Besinnung gezwungen. In der Erfahrung des Krankseins kann sich die Chance eröffnen, scheinbar Selbstverständliches wie Gesundheit und Leistungsfähigkeit als nicht selbstverständlich zu erkennen, die Unsicherheit, Begrenztheit, die Zerbrechlichkeit unserer Existenz und aller unserer Hervorbringungen wahrzunehmen und mit diesen kostbaren Gaben wertschätzender und achtsamer umzugehen.

Es ist anzuerkennen, dass die Ausrichtung der Neuzeit auf Spezialisierung und Quantifizierung generell und speziell im medizinischen Bereich große Erfolge ermöglicht hat. Andererseits wird die eindimensionale Quantifizierung unter den Etiketten „Rationalisierung“, „Optimierung“", „Effizienz-Steigerung“, „Qualitätssicherung“ ... zunehmend auf alle Lebensbereiche, auch auf Medizin und Pflege ausgeweitet. Alles wird auf das Quantifizierbare und Digitalisierbare, auf „Effizienz“ und letztlich auf „Kapitalerträge“ ausgerichtet. Das Nicht-Quantifizierbare, Unverzweckbare, Freie, Schöne, Geschenkhafte, Unkaufbare und Unverkäufliche finden dadurch zunehmend weniger Raum und Aufmerksamkeit. Wenn diese „Rationalität“ und „Effizienz“ unser Leben, unsere Zivilisation, unser Medizinsystem immer stärker bestimmen, dann werden im gleichen Ausmaß die Menschen auf produzierende und konsumierende Rädchen in einer gesellschaftlichen Maschinerie, auf Kostenfaktoren und Kalkulationsgrößen reduziert. Die Konsequenzen sind ein Verkümmern wesentlicher personaler Qualitäten, zunehmende Beziehungs-Unfähigkeit, „Hirnund Herz-Lähmung“, eine geistige, seelische und kulturelle Verarmung mitten im materiellen Wohlstand!

Unsere Zivilisation ist total auf das Leben hier und jetzt fixiert. Man hat grenzenlose Erwartungen an dieses Leben und verdrängt alles, was dies in Frage stellen könnte. Das Unkalkulierbare und Unverfügbare, unüberwindliche Einschränkungen, am extremsten der Tod, sind solche Infragestellungen, die man weithin verdrängt. Viele Maßlosigkeiten und deformierte Lebensmuster in unserer Zivilisation sind unschwer als Kompensationen dieser verdrängten Fragen zu dechiffrieren. Nachdenkliche Menschen beschleicht zunehmend eine tiefe Skepsis hinsichtlich der Sinnhaftigkeit dieser gesellschaftlichen Dynamik und solcher Fortschrittskonzepte. Man fragt wieder stärker über das Materielle, über alles Quantifizierbare hinaus: Gibt es Sinn und tragfähige Hoffnung in meinem Leben, für unsere Welt, über alles vordergründige Getriebe, auch über den Tod hinaus? Die großen religiösen Traditionen artikulieren Antworten auf die Fragen nach einer transzendenten Herkunft und $\mathrm{Zu}-$ kunft der Menschen. Diese Antworten sind in 
ihren Inhalten und Konsequenzen nicht gleich: „Transzendenz" kann die Erdenwirklichkeit sowie Wert und Würde konkreter Menschen bis zur Wertlosigkeit relativieren, wie in bestimmten esoterischen und fernöstlichen Traditionen, oder durch Erweiterung der Perspektive über die Phase in Raum und Zeit hinaus bestärken und begründen. Für die biblisch-christliche Sicht des menschlichen Lebens ist eine nüchterne Hoffnung zentral: Welt und Menschen stammen aus der schöpferischen Zuwendung Gottes. Auf den oft schwierigen Wegen des Lebens ist $\mathrm{zu}$ entdecken und $\mathrm{zu}$ erlernen, wie menschenwürdig gelebt werden kann. Auf den geschichtlichen Wegen der Menschheit sind Welt und Gesellschaft schrittweise zu kultivieren. Nach biblischer Orientierung gelingen die Entfaltung des Lebens, Befreiung und Heilung letztlich nicht durch „Besitzen und Beherrschen“, sondern in „Beziehung“ und es gibt Hoffnung über den Tod hinaus.

Viele Menschen spüren mehr oder minder deutlich eine Erschöpfung der neuzeitlichen Orientierungen und Hoffnungen. In diesem Zusammenhang nimmt auch die Skepsis gegenüber einer einseitig wissenschaftlich-technisch-ökonomisch geprägten Medizin zu. Ich beteilige mich mit diesen Überlegungen nicht an einer oft billigpolemischen Geringschätzung der Neuzeit und der großen Errungenschaften moderner Medizin, vielmehr geht es, aufbauend auf diesen groBen Leistungen, um sinnvolle Neuorientierungen. So verständlich und notwendig das neuzeitliche Setzen auf „Entgrenzung“ war, jetzt ist es an der Zeit, von illusionärer Grenzenlosigkeit zu lassen und sich auf eine bewusste Kultivierung des Begrenzten zu konzentrieren. Galten neuzeitlich Grenzen als „zu überwinden“, galt das Begrenzte als minder, so ist jetzt wahrzunehmen, dass alles auf Erden begrenzt ist. Wichtig ist eine qualitative Interpretation dieser faktischen Feststellung: Das Begrenzte ist neu zu verstehen als einzigartig und kostbar. Um das am Beispiel von Wasser zu veranschaulichen: Solange es unbegrenzt verfügbar ist, schätzt man es kaum, erst wenn es knapp wird, lernt man es schätzen und geht damit sorgfältig um -, in der Wüste ist Wasser kostbarer als Gold. Analog gilt dies für alle materiellen Ressourcen und, auch für Zeit, Kraft, Aufmerksamkeit, für die Tage und Jahre unseres Lebens. All das ist begrenzt und deshalb einzigartig und schätzens- wert. Diese Einsicht kann und soll einen kultivierten, wertschätzenden Umgang mit dem Begrenzten stimulieren.

Weiters ist bei aller Anerkennung der Errungenschaften durch die neuzeitliche Spezialisierung jetzt eine stärkere Beachtung der $\mathrm{Zu}$ sammenhänge in der Medizin, mit Lebenswelt und Lebensstil, mit Vorsorge und Nachsorge angezeigt. Es ist sehr zu beachten, dass sich gegenüber früheren Verhältnissen die Arten und Ursachen der Erkrankungen sehr stark verändert haben: Den größten, folgenschwersten und aufwändigsten Anteil bilden mittlerweile die Zivilisationskrankheiten. Eine nachhaltige $\mathrm{Ab}$ hilfe bringt da nicht eine weitere Steigerung der Investitionen in das Medizinsystem, sondern eine Veränderung unserer Lebensmuster, persönlich und öffentlich: Etablierung entsprechender Rhythmen von Aktivität/Anspannung und kontemplativen Phasen mit zur Ruhe Kommen, Rückblick und Ausblick, bessere Prioritäten und Proportionen in der Lebenskultur, Überwindung pathogener innerer und äußerer Unterund Überspannungen, eine stärkere Beachtung personaler Aspekte, Beachtung der heilsamen wie krankmachenden „Beziehungen“, der SinnFragen im persönlichen und öffentlichen Leben.

Um abschließend zusammenzufassen: Als denk- und entscheidungsfähige Menschen haben wir in unseren unübersichtlichen Hochleistungsgesellschaften beim Umgang mit Leben generell und speziell im medizinischen Bereich große Möglichkeiten und eine hohe Verantwortung. Besonders wichtig und schwierig ist ein kultivierter Umgang mit Geld und Macht, in der Gesellschaft allgemein und besonders im medizinischen Bereich. Wir müssen sehr aufpassen, dass wir nicht schleichend am Anfang, am Ende und mitten im Leben unmenschliche Wertungen praktizieren: Haben nur leistungsfähige Menschen mit genug Geld Geltung und Lebensrecht? Wer hat keine Schwächen, Handikaps und Grenzen? Wer definiert „normal“? Was ist mit uns, wenn wir älter und schwächer werden? Derartige Entscheidungen, die oft über Lebensqualität, Anerkennung oder Marginalisierung, nicht selten über Leben und Tod entscheiden, darf man nicht individualistischer Willkür, rein technokratisch-ökonomischen Maßstäben, sozialdarwinistischen Standards überlassen!

Es geht um das Gelingen des Lebens. Physiotherapeuten können mit ihrem fachlichen 
Können und ihrem persönlichen Engagement dazu Wertvolles beitragen. Gewiss muss man in hochentwickelten medizinischen Systemen mit vielen Vorgaben zurechtkommen, mit notwendigen, sinnvollen und problematischen. Wichtig ist, dass über allen sachlich-fachlichen Fakten und Vorgaben das kostbare und fragile Menschliche nicht übersehen, sondern aufmerksam wahrgenommen wird -, das Menschliche, das auch Physiotherapeuten bei aller fachlichen Kompetenz nie voll ,in den Griff kriegen“, wofür sie aber viel Heilsames beitragen können.

\section{LITERATUR}

ANZENBACHER, A.: Einführung in die Ethik, Düsseldorf, ${ }^{3} 2003$.

AUFDERHEIDE, D., DABROWSKI, M. (Hg.): Gesundheit - Ethik - Ökonomik. Wirtschaftsethische und moralökonomische Perspektiven des Gesundheitswesens, Berlin, 2002.

BARMEYER, J.: Praktische Medizinethik: die moderne Medizin im Spannungsfeld zwischen naturwissenschaftlichem Denken und humanitärem Auftrag. Münster, ${ }^{2} 2003$

BONDOLFI, A.: Ethisch denken und moralisch handeln in der Medizin. Anstöße zur Verständigung, Zürich, 2000. CAMERON, M., H.: Physical Agents in Rehabilitation. From Research to Practice, Philadelphia: PA, ${ }^{2} 2003$.

CHERRY, M. J.: Persons and their bodies. Rights, responsibilities, relationships, Dordrecht, 1999.

FREIDL, C.: Wellnessboom. Erholung - oder zu viel des Guten?, Saarbrücken, 2004.

GORDIJN, B., HAVE, H. ten (Hg.): Medizinethik und Kultur, Stuttgart, 2000.

GRAUMANN, S. et al. (Hg.): Ethik und Behinderung: Ein Perspektivenwechsel, Frankfurt-New York, 2004.

HARRIS, J. (Hg.): Biomedical Ethics, Oxford, 2001

HASLBECK, B., GÜNTHER, J. (Hg.): Wer hilft, wird ein anderer. Zur Provokation christlichen Helfens. Festschrift Isidor Baumgartner: Berlin, 2006.

HONNEFELDER, L., RAGER, G. (Hg.): Ärztliches Urteilen und Handeln. Zur Grundlegung einer medizinischen Ethik, Frankfurt, 1994.

HÜTER-BECKER, A. (Hg.): Das Neue Denkmodell in der Physiotherapie, Bd.1: Bewegungssystem, Stuttgart, ${ }^{2} 2006$

HÜTER-BECKER, A..: Der Paradigmenwechsel in der Physiotherapie und das Bobath-Konzept Gesamt. In Krankengymnastik. Zeitschrift für Physiotherapeuten, 52 (2000). s. 277-282.

ILLHARDT, F.-J.: Ethik in der Schmerztherapie. Zentrum für medizinische Ethik: Bochum, 2000

KODALLE, K.-M. (Hg.): Homo perfectus? Behinderung und menschliche Existenz, Würzburg, 2004.

KOOL, J., de BIE, R.: Der Weg zum wissenschaftlichen Arbeiten. Ein Einstieg für Physiotherapeuten: Stuttgart 2000 .
KORFF, W.: Wie kann der Mensch glücken? Perspektiven der Ethik, München, 1985.

KÖRTNER, U. H. J.: Unverfügbarkeit des Lebens? Neukirchen-Vluyn, 2001.

KÖRTNER, U. H. J.: Grundkurs Pflegeethik, Wien, 2004. KRESS, H.: Medizinische Ethik. Kulturelle Grundlagen und ethische Wertkonflikte heutiger Medizin, Stuttgar,t 2003.

LENK, Ch.: Therapie und Enhancement: Ziele und Grenzen der modernen Medizin, Münster, 2002.

MAIO, G.: Ethik der Forschung am Menschen. Medizin und Philosophie, Bd. 6, Stuttgart, 2001.

MEISSER-PÖTHIG, D.: Anleitung zum Krankwerden oder Ist Gesundheit heilbar?, Treuchtlingen, 1999.

MIETH, D.: Kleine Ethikschule, Freiburg, 2004.

MORRIS, D. B.: Krankheit und Kultur-Plädoyer für ein neues Körperverständnis, München, 2000.

OBERENDER, P., ECKER, T.: Grundelemente der Gesundheitsökonomie, Bayreuth, 2001.

O'MAHONY, P.: Nature, risk, and responsibility. Discourses of biotechnology, Basingstoke, 1999.

PÖLTNER, G.: Grundkurs Medizin-Ethik, Wien, 2002.

REIBNITZ, Ch., SCHNABEL, P.-E., HURRELMANN, K. (Hg.): Der mündige Patient - Konzepte zur Patientenberatung und Konsumentensouveränität im Gesundheitswesen, München, 2001.

RENÖCKL, H.: Ernstfall Organtransplantation. Ethische Aspekte gegen die Verdrängung des Pathischen, in: Haslbeck, B., Gunter, J. (Hg.): Wer hilft, wird ein anderer. Festschrift Isidor Baumgartner: Berlin, 2006. s. 197-209.

RICE, T., S.: Gesundheitsökonomie. Eine kritische Auseinandersetzung, Bonn, 2004.

RICKEN, F.: Allgemeine Ethik, Stuttgart, ${ }^{4} 2003$.

RIEDER, K.: Dialogische Dienstleistungsarbeit in der Krankenpflege: Konzepte für die Analyse von Belastungen und Anforderungen, in: Ulich, E. (Hg.): Arbeitspsychologie in Krankenhaus und Arztpraxis. Arbeitsbedingungen, Belastungen, Ressourcen, BernGöttingen-Toronto-Seattle, 2003.

RYCHLIK, R.: Gesundheitsökonomie. Gesundheit und Praxis, Stuttgart, 1999

SCHLANDER, M.: Gibt es einen normativen Anspruch der Gesundheitsökonomie? in: Kick, H., A.., Taupitz, J. (Hg.): Gesundheitswesen zwischen Wirtschaftlichkeit und Menschlichkeit, Münster, 2005. s. 37-112.

SCHMID, W.: Schönes Leben? Einführung in die Lebenskunst, Frankfurt, ${ }^{2} 2005$.

SCHMIDBAUER, W.: Hilflose Helfer - Über die seelische Problematik helfender Berufe, Reinbek, 1997.

SCHOCKENHOFF, E.: Ethik des Lebens. Ein theologischer Grundriss, Mainz, ${ }^{3} 2000$.

SCHRÖDER, H., HACKHAUSEN, W. (Hg.): Persönlichkeit und Individualität in der Rehabilitation, Frankfurt, 2001.

STÖHR, M.: Die Wahrheit über Anti-Aging. Risiken erkennen - Chancen nutzen, Berlin, 2005.

WIESEMANN, C.: Medizinethik, Stuttgart, 2004

ZILLESSEN, A., HEIN, R.: Marketing für die Physiotherapie. Erfolg durch Corporate Identity, Stuttgart, 2005. 


\section{Autoren-Information:}

Univ.-Prof. Dr. Helmut Renöckl, in Linz: Honorarprofessor für ,Ethik in Naturwissenschaften und Technik" an der J. Kepler Universität, Lehrauftrag „Ethik“ an der Kath. Theolog. Privatuniversität, Leiter des „Instituts für konkrete Ethik und ethische Bildung“". In České Budějovice/Budweis: Leiter des Lehrstuhls für „Theologische Ethik, Sozialethik und ethische Bildung“ und Vorstand des „Instituts für Wirtschaftsethik und Regionalentwicklung“ an der Südböhmischen Universität. Aktivitäten im Bereich Medizinethik: Seit 1984 leitende Mitarbeit bei medizinethischen Aus- und Weiterbildungsaktivitäten für Ärzte, Pflege- und therapeutische Berufe, Mitglied der Medizinethik-Arbeitsgruppe der OÖ. Medizinischen Gesellschaft. Mitglied der „Europäischen Akademie der Wissenschaften und Künste" und weiterer internationaler wissenschaftlicher Vereinigungen. Rund 140 Publikationen.

\section{Údaje o autorovi:}

Univ.-Prof. Dr. Helmut Renöckl, v Linci: čestná profesura pro „etiku v prrírodních vědách a technice“ na Keplerově univerzitě, vyučující etiky na Katolické teologické soukromé univerzitě, ředitel Institutu pro konkrétní etiku a etické vzdělávání. V Českých Budějovicích: vedoucí katedry teologické etiky, sociální etiky a etického vzdělávání a ředitel Institutu pro hospodářskou etiku a regionální rozvoj Jihočeské univerzity. Aktivity v oblasti lékařské etiky: od roku 1984 vedoucí spolupráce při lékařsko-etických aktivitách vzdělání a dalšího vzdělávání lékařuo, terapeutů a ošetřujícího personálu, člen pracovní skupiny pro lékařskou etiku Hornorakouské lékařské společnosti. Člen Evropské akademie věd a umění a dalších mezinárodních vědeckých svazů. 140 publikací.

\author{
Helmut Renöckl \\ helmut.renoeckl@liwest.at \\ renoeckl@fim.uni-linz.ac.at
}

